\title{
New perspective for nutritional support of cancer patients: Enteral/parenteral nutrition
}

\author{
GAMZE AKBULUT \\ Department of Nutrition and Dietetics, Faculty of Health Sciences, Gazi University, Besevler, Ankara, Turkey
}

Received January 20, 2011; Accepted March 24, 2011

DOI: 10.3892/etm.2011.247

\begin{abstract}
Cancer and its treatment result in severe biochemical and physiological alterations associated with a deterioration of quality of life (QoL). Cancer-related malnutrition may evolve into cancer cachexia due to complex interactions between proinflammatory cytokines and the host metabolism. Depending on the type of cancer treatment (either curative or palliative), the clinical condition of the patient and nutritional status, adequate and patient-tailored nutritional intervention should be prescribed (diet counseling, oral supplementation, enteral or total parenteral nutrition). Nutritional support has been widely advocated as adjunctive therapy for a variety of underlying illnesses, including surgery and medical oncotherapy (radiation or chemotherapy for cancer). Glutamine, n-3 fatty acids and probiotics/prebiotics are therapeutic factors that potentially modulate gastrointestinal toxicity related to cancer treatments. Enteral and parenteral nutrition may help improve patient survival, functional status and QoL, yet the benefits appear to be primarily limited to patients with good functional status and with gastrointestinal disease affecting nutritional intake. Parenteral nutrition offers the possibility of increased or maintenance of the nutrient intake in patients for whom normal food intake is inadequate and for whom enteral nutrition is not feasible, is contraindicated or is not accepted by the patient. This article reviews evidence on issues relevant to enteral and parenteral nutrition in patients with cancer.
\end{abstract}

\section{Contents}

1. Introduction

2. Cancer cachexia

3. Nutritional support for cancer patients

4. Conclusion

Correspondence to: Dr Gamze Akbulut, Department of Nutrition and Dietetics, Faculty of Health Sciences, Gazi University, Besevler, Ankara, Turkey

E-mail: dytgamzea@gmail.com

Key words: cancer, cachexia, enteral/parenteral nutrition, n-3 fatty acids, glutamine, prebiotic-probiotic oligosaccharides

\section{Introduction}

Malnutrition is commonly observed in cancer patients and adversely affects the quality of life (QoL) and survival of these patients. It is caused by a variety of factors, including decreased food intake, adverse effects from anticancer treatment and wasteful metabolic processes (1). Over the past two decades, there have been major advances in the methods and techniques used in the dietary therapy of patients with cancer and other diseases. Enteral nutrition is developing rapidly as endoscopic techniques have made it simpler to place feeding tubes, and a variety of enteral nutrition solutions are commercially available. Enteral nutrition is an effective way to deliver nutrients when patients are unable to ingest food because of neurologic disorders or structural abnormalities in the upper gastrointestinal tract, including the oropharynx, esophagus and stomach. The role of enteral nutrition as an adjuvant to anticancer therapy has not been fully evaluated. Glutamine, n-3 polyunsaturated fatty acids (FAs) and probiotics/prebiotics are therapeutic factors that potentially modulate gastrointestinal (GI) toxicity related to cancer treatments (2).

Total parenteral nutrition (TPN) is an effective method of delivering nutrients into the blood stream. It has been proven to be life-saving for patients with chronic severe gastrointestinal insufficiency (such as short bowel or radiation enteritis), whose cancer is cured or non-progressive. As an adjuvant to chemotherapy, TPN does not appear to be useful, unless there are prolonged periods of gastrointestinal toxicity (as in the case with bone marrow transplantation) that severely limit oral intake and absorption (3). Perioperative parenteral nutrition is only recommended in malnourished patients when enteral nutrition is not feasible. In non-surgical well-nourished oncologic patients, routine parenteral nutrition is not recommended since it has been proven to offer no advantage and is associated with increased morbidity. A benefit, however, has been reported in patients undergoing hematopoietic stem cell transplantation (HSCT). Short-term parenteral nutrition is commonly accepted in patients with acute gastrointestinal complications from chemotherapy and radiotherapy, and long-term (home) parenteral nutrition may sometimes be a life-saving maneuver in patients with subacute/chronic radiation enteropathy (4).

Weight loss, decreased appetite and difficulty in the consumption of food are common features of the terminal phase of cancer. Some patients also become physically unable to take in sufficient nutrition, or eating may become painful, 
time-consuming or otherwise burdensome. Difficulty eating may be self-limited, such as temporary nausea or illness, or may be expected to last the rest of the lifespan, such as untreatable gastrointestinal obstruction. Treatment of underlying symptoms or conditions, changes in diet and nutritional supplements may be helpful in certain situations, and appetite stimulants may increase intake, body weight and QoL, but they do not affect the prognosis in the terminally ill (5).

\section{Cancer cachexia}

Cancer cachexia is a complex syndrome characterized by a chronic, progressive, involuntary weight loss which is poorly or only partially responsive to standard nutritional support and it is often associated with anorexia, early satiety and asthenia. It is usually attributable to two main components: a decreased nutrient intake (which may be due to critical involvement of the gastrointestinal tract by the tumor, or to cytokines and similar anorexia-inducing mediators); and metabolic alterations due to the activation of systemic proinflammatory processes (4).

Metabolic derangements may result in insulin resistance, increased lipolysis and normal or increased lipid oxidation with loss of body fat, increased protein turnover with loss of muscle mass and an increase in the production of acute phase proteins. The systemic inflammatory reaction that develops with many cancers is an important cause of loss of appetite (anorexia) and weight (6). The syndrome of decreased appetite, weight loss, metabolic alterations and an inflammatory state is therefore referred to as cancer cachexia or cancer anorexiacachexia syndrome. These cytokine-induced metabolic alterations appear to prevent cachectic patients from regaining body cell mass during nutritional support, are associated with a reduced life expectancy and are not relieved by exogenous nutrients alone (7-9).

Cancer patients traditionally have been regarded as hypermetabolic; however, a heterogeneous picture of energy expenditure has been described, with resting energy expenditure ranging from less than $60 \%$ to more than $150 \%$ of that predicted (10). Although cancer patients often have reduced food intake (due to systemic effects of the disease, local tumor effects, psychological effects or adverse effects of treatment), alterations in nutrient metabolism and resting energy expenditure may also contribute to the nutritional status (11). Whereas resting energy expenditure is increased, total energy expenditure may be unchanged due to a decrease in physical activity. Thus, overall energy balance may be maintained by a concomitant reduction in activity, while this decreased physical activity may be a reflection of a reduced QoL (12).

Cachexia cannot be easily differentiated from undernutrition due to simple starvation. Both cachectic and undernourished patients exhibit a loss of body weight and may be anorectic; however, simply undernourished patients show a tendency to save their protein mass, they decrease their resting energy expenditure and they respond quite well to nutritional support if their general status is not compromised in an irreversible way (Fig. 1) (13).

Anticancer treatments can also be a major cause of malnutrition. Chemotherapy causes nausea, vomiting, abdominal cramping and bloating, mucositis, paralytic ileus and even malabsorption. Despite the recent advent of antiemetic drugs, such as the setrons, and the optimization of their administration schedule, vomiting remains an important cause of malnutrition in cancer. Various antineoplastic agents, such as fluorouracil, adriamycin, methotrexate and cisplatin, induce severe gastrointestinal complications (14).

Health-related QoL is a multidimensional concept which quantifies the psychological, physical and social effects of an illness and its therapy. In cancer patients, the health status is well reflected in the measured QoL, which is largely influenced by nutritional aspects. The evaluation of QoL assesses patient well-being by taking into account physical, psychological and social conditions (15). Cancer and its treatment result in severe biochemical and physiological alterations associated with a deterioration of QoL. These metabolic changes lead to decreased food intake and promote wasting. Above and beyond the physical and the metabolic effects of cancer, patients also suffer from psychological distress, including depression. Depending on the type of cancer treatment (either curative or palliative), the clinical condition of the patient and nutritional status, adequate and patient-tailored nutritional intervention should be prescribed (diet counseling, oral supplementation, enteral or total parenteral nutrition). Such an approach, which should be started as early as possible, reduces or even reverses their poor nutritional status, improves their performance status and consequently their QoL. Nutritional intervention accompanying curative treatment has an additional and specific role, which is to increase the tolerance and response to oncology treatment, decrease the rate of complications and possibly reduce morbidity by optimizing the balance between energy expenditure and food intake. In palliative care, nutritional support aims at improving patient QoL by controlling symptoms, such as nausea, vomiting and pain related to food intake, and by postponing loss of autonomy. Assessment of QoL should be considered in the evaluation of any nutritional support to optimize its benefits in regards to the needs and expectations of the patient (16).

Interactions between QoL and nutritional status of the patients. The inadequate nutritional status and cancer anorexia-cachexia syndrome related to it are clinically relevant since the response to antineoplastic measures, such as radiation and chemotherapy, may be diminished, their side effects aggravated and patient QoL and prognosis negatively affected. Therefore, supportive nutritional care of oncological patients is of central importance (17). An impaired nutritional status is associated with reduced QoL, lower activity level, increased treatmentrelated adverse reactions, reduced tumor response to treatment and reduced survival. However, a cause-effect relationship is yet to be established (18).

A link between QoL and nutritional status is supported by evidence that an insufficient nutritional status is frequently related to reduced QoL $(19,20)$. Similarly, food intake - one of the major determinants of nutritional status - appears to influence QoL, as a correlation between them exists (21). Moreover, a low QoL is associated with nutrition-related symptoms and weight loss (22).

Influence of nutritional intervention on oncology treatment. In curative oncology treatment, nutritional intervention aims to reduce the number of complications and to shorten the 




Figure 1. Monitoring of cachexia.

recovery phase. The probability of developing malnutrition is increased after curative treatment as aggressive therapeutic strategies are frequently used. A prolonged therapy based on patient response may further aggravate the risk for impaired nutritional status, although it should be acknowledged that tumor site and inherent nutritional risk of the treatment also contribute to the deterioration of the nutritional status. Nevertheless, alleviation of nutrition-related symptoms and signs may contribute to the well being of the patient $(23,24)$.

In palliative oncology treatment, the aim of nutritional intervention is to sustain or enhance recovery of patient performance in everyday life, their well-being and their QoL. Palliative cancer treatment is also a system of care that strives to relieve the suffering of patients with progressive cancer. Given the intractable symptoms with which certain malignancies manifest, palliative care offers a practical approach towards improving patient QoL. However, there is an array of ethical issues associated with this treatment strategy, such as particular methods of pain relief, a reliable assessment of suffering, autonomy and multi-specialist care (25). The development of palliative care in terms of recognizing the needs of the dying has becoming a nursing and medical speciality. The involvement of the World Health Organization (WHO) in palliative care and the continuous development of treatment modalities available to cancer patients creates the expectation that outcomes for the patient should also be positively influenced $(26,27)$. Palliative care is focused on maintaining adequate hydration, alleviating or controlling symptoms (e.g., nausea and vomiting) and maintaining body weight and composition. When selecting the type of nutritional intervention, for instance oral nutritional supplementation (ONS), enteral nutrition or parenteral nutrition, the wishes of the patient and their family must be considered (28).

\section{Nutritional support for cancer patients}

The purpose of nutritional assessment is to identify the subset(s) of patient who may benefit from dietary counseling by a dietitian, to determine the severity and cause(s) of malnutrition, to identify patients at risk of complications of chemotherapy, radiation therapy or surgery, and to assess the efficacy of nutritional support. The nutritional parameters and indices should have sufficient sensitivity and specificity to reliably reflect the course of malnutrition during the disease, from baseline at diagnosis to remission or cure, through each specific therapeutic intervention. Nutritional assessment must be combined with a careful evaluation of performance status and QoL, so that nutritional management is correctly adapted to the patient's real needs and entails a minimum of constraints. Ideally, nutritional support should benefit the patient without feeding the tumor or, better, while starving the tumor. Drastic restriction of the amount of protein in food inhibits tumor growth in most animal models, but limitation of protein intake is also detrimental to a malnourished host. The stimulation of tumor growth by enteral or parenteral nutrition has never been clearly demonstrated in humans. In vivo evaluation of tumor growth is technically difficult, and most studies rely on data gathered in very small populations $(14,23,29)$.

Enteral nutrition in non-surgical cancer patients. Enteral nutrition (EN) by means of ONS and tube feeding (TF) offers the possibility of increasing or ensuring nutrient intake in cases where normal food intake is inadequate. There are no data from controlled studies to suggest a cancer-specific enteral formula. Standard formulas are recommended for EN of cancer patients. General information about enteral nutrition in shown in Table I (30).

The therapeutic goal for cancer patients is the improvement of function and outcome by i) preventing and treating undernutrition; ii) enhancing antitumor treatment effects; iii) reducing adverse effects of antitumor therapies; and iv) improving QoL (30).

Nutritional therapy should be initiated when undernutrition already exists or when it is anticipated that the patient may be unable to eat for more than 7 days. EN should also be initiated when an inadequate food intake $(<60 \%$ of estimated energy expenditure) is anticipated for more than 10 days. It should substitute the difference between actual intake and calculated requirements.

According to recent guidelines, initiating EN in patients is indicated upon decreased oral intake $(31,32)$. When nutritional intake is chronically reduced, then a corresponding weight loss and a worsening of prognosis are anticipated. To determine a reduced intake of normal food, a simple $24 \mathrm{~h}$ recall is usually adequate. If this proves difficult in individual cases, it may be appropriate to ask the patient whether his/her nutritional intake is less than $50 \%$ (low intake) or less than 25\% (minimal intake) of their usual intake before the onset of the disease. In patients who are losing weight due to insufficient nutritional intake, EN should be provided to improve or maintain nutritional status (31). This may also contribute to the maintenance of QoL (30).

Omega-3 fatty acids. Omega-3 (n-3) FAs are long-chain polyunsaturated FAs with a final carbon-carbon double bond in 
Table I. Summary of information concerning enteral nutrition: Non-surgical oncology.

\begin{tabular}{|c|c|}
\hline Subject & Recommendations \\
\hline General & $\begin{array}{l}\text { Nutritional assessment of cancer patients should be performed frequently, and nutritional } \\
\text { intervention initiated early when deficits are detected. }\end{array}$ \\
\hline $\begin{array}{l}\text { General } \\
\text { indications }\end{array}$ & $\begin{array}{l}\text { There are no reliable data that show any effect of enteral nutrition on tumor growth. Such } \\
\text { theoretical considerations should, therefore, have no influence on the decision to feed a } \\
\text { cancer patient. Start nutritional therapy if undernutrition already exists or if it is anticipated } \\
\text { that the patient will be unable to eat for }>7 \text { days. Start enteral nutrition if an inadequate food } \\
\text { food intake ( }<60 \% \text { of estimated energy expenditure for }>10 \text { days) is anticipated. It should } \\
\text { substitute the difference between actual intake and calculated requirements. }\end{array}$ \\
\hline
\end{tabular}

Perioperative

During radiotherapy or radiochemotherapy

During

chemotherapy

During stem cell

transplantation

In incurable

patients

Application

Route

During radiotherapy or radiochemotherapy

Type of formula General

Perioperative

During stem cell transplantation

Drug treatment
In weight-losing patients, due to insufficient nutritional intake, enteral nutrition should be provided to improve or maintain nutritional status. Patients with severe nutritional risk may benefit from nutritional support 10-14 days prior to major surgery, even when surgery has to be delayed.

Use of intensive dietary advice and oral nutritional supplements is advised to increase dietary intake and prevent therapy-associated weight loss and interruption of radiation therapy.

Routine enteral nutrition is not indicated during radiation therapy. Routine enteral nutrition during chemotherapy has no effect on tumor response to chemotherapy or on chemotherapy-associated unwanted effects and, therefore, is not considered useful.

Routine use of enteral nutrition is not recommended.

When oral intake is decreased, parenteral nutrition may be preferred to tube feeding in certain situations (i.e., increased risk of haemorrhage and infections associated with enteral tube placement in immunocompromised and thrombocytopenic patients). Enteral nutrition may be provided in order to minimize weight loss, as long as the patient consents and the dying phase has not started. When the end of life is very close, most patients only require minimal amounts of food and little water to reduce thirst and hunger. Small amounts of fluid may also help to avoid states of confusion induced by dehydration.

Subcutaneously infused fluids in hospital or at home may be helpful and may also provide a vehicle for the administration of drugs. The enteral route is preferable whenever feasible. Administration of pre-operative enteral nutrition is preferable before admission to the hospital.

Use of tube feeding is advocated when an obstructing head or neck or esophageal cancer interferes with swallowing, or when severe local mucositis is expected.

Tube feeding is either delivered via transnasal or percutaneous routes. Because of radiation-induced oral and esophageal mucositis, a percutaneous gastrostomy may be preferred.

Use of standard formulae is advised. Regarding n-3 fatty acids, randomized clinical trial evidence is contradictory/controversial and at present it is not possible to reach any definite conclusion with regard to improved nutritional status/physical function. It is unlikely that n-3 fatty acids prolong survival in advanced cancer.

Use of pre-operative enteral nutrition preferably with immunomodulating substrates (arginine, n-3 FAs, nucleotides) is advisable for 5-7 days in all patients undergoing major abdominal surgery independent of their nutritional status.

Enteral administration of glutamine or eicosapentanoic acid is not recommended due to inconclusive data.

In the presence of systemic inflammation, pharmacological efforts are recommended in addition to nutritional interventions to modulate the inflammatory response. In cachectic patients, steroids or progestins are recommended in order to enhance appetite, modulate metabolic derangements and prevent impairment of QoL. Administration of steroids for short-term periods should be carried out only weighing their benefits against their adverse side effects. Consider the risk of thrombosis during progestin therapy. 
the $n-3$ position, the third bond, from the methyl end of the chain. Omega-6 (n-6) FAs have a similar structure with the first double bond 6 carbons from the methyl end of the chain. Humans are unable to desaturate the n-3 or n- 6 double bond and as such this makes both compounds 'essential FAs' which can only be obtained from dietary sources. Omega-6 fatty acid is consumed as linoleic acid or arachidonic acid found in meats and vegetable oils (safflower, corn and soybean oil). The principal dietary source of n-3 FAs is from oily cold-water fish namely eicosapentaenoic acid (EPA) and docosahexaenoic acid (DHA). General interest has focused on the properties of n-3 FAs. Both omega-3 and omega- 6 FAs are used as substrates for the production of eicosanoids which are a class of compounds, including prostaglandins (PGs), thromboxanes and leukotrienes intimately involved in immunomodulation, inflammation and tumor formation. Eicosanoids produced using n-6 FAs (arachidonic acid) as a substrate stimulate inflammation and tumor angiogenesis, whereas eicosanoids produced from n-3 FAs, EPA and DHA are anti-inflammatory and do not stimulate angiogenesis $(33,34)$. The administration of fish oil reduces production of cytokines, such as IL-6, TNF and IL-1, in healthy subjects (35).

The potential benefit of n-3 FA supplementation is the effect of these fats on cachexia. Anti-inflammatory agents, such as fish oil, in combination with nutritional supplementation may reverse features of cachexia (36). Pancreatic cancer patients often develop debilitating cachexia. Certain studies have shown weight gain and improved QoL after daily supplementation of the diet with an energy- and protein-dense (610 kcal, $32.2 \mathrm{~g}$ protein) liquid supplement containing $2.2 \mathrm{~g}$ EPA and $0.96 \mathrm{~g}$ DHA for 8 weeks (37). Patients who consumed a supplement that contained energy and protein, but did not contain the n-3 FAs, did not gain weight.

The effects of omega-3 FAs on improvement in appetite and body weight have been studied in cancer patients (30). Takatsuka et al (38) studied 16 consecutive patients, 7 of whom received $1.8 \mathrm{~g}$ /day EPA orally from 30 days before until 180 days after allogeneic HSCT. EPA was found to lower the levels of prostanoids and cytokines. In addition, complications of HSCT were less and the survival rate was significantly higher in the group treated with EPA. In a short-term (2-week) placebo-controlled trial, Bruera et al (39) studied 60 weightlosing cancer patients and found no effect of $1.8 \mathrm{~g} \mathrm{EPA} /$ day on appetite, tiredness, nausea, well-being, caloric intake and nutritional status or function. However, it was indicated that ingested EPA accumulates in tissues over time, and 2 weeks may be too short to induce clinically measurable effects. In a clinical study, a group of 18 weight-losing patients with advanced pancreatic cancer received oral fish-oil preparations providing approximately $2.2 \mathrm{~g}$ of EPA and $1.4 \mathrm{~g}$ of the related docosahexanoic acid daily. Before treatment, all patients experienced weight loss at a median rate of $2.9 \mathrm{~kg} / \mathrm{month}$. After 3 months of supplementation, the patients' weights stabilized, with less than half of the patients continuing to lose weight. There was no change in the percentage of total body water during the study, suggesting that patients were not simply retaining fluid (40).

The use of EPA-containing protein- and energy-dense ONS (EPA-ONS) was shown to reduce weight loss, increase lean body mass (LBM), improve functional capacity, nutri- tional status and QoL, however not to any greater degree than conventional supplements (41). Intention to treat patients indicated that, at the mean dose taken, enrichment with n-3 FAs did not provide a therapeutic advantage and that both supplements were equally effective in arresting weight loss. Post hoc dose-response analysis suggests that if taken in sufficient quantity, only the n-3 FA-enriched energy- and protein-dense supplement results in net gain of weight, lean tissue and improved QoL. (42). Read et al (43) indicated that after an average amount of $408 \mathrm{ml} /$ day EPA for 3 weeks, a significant increase was noted in mean patient weight $(2.5 \mathrm{~kg})$ $(\mathrm{p}=0.03)$, and LBM was maintained. In the summary of this study, it was stated that dietary counseling by suitably qualified dietitians and the administration of EPA in patients with advanced colerectal cancers receiving chemotherapy, help to maintain weight and possibly improve symptom control, nutritional status and QoL.

In summary, multiple mechanisms play a role in the suppression of tumor growth by n-3 FAs. Some of the mechanisms may play a more dominant role in particular tumor types, i.e., alteration of estrogen is likely to be more important for suppression of breast than of esophageal cancer. Pre-clinical studies indicate that n-3 FAs should be beneficial for cancer treatment. Mechanistic studies indicate feasible mechanisms for the influence of n-3 FAs on tumor growth, survival and response to chemotherapy. A limited number of clinical studies indicate that n-3 FAs may be beneficial when consumed before chemotherapy. It seems important to commence human trials using an (n-3) fatty acid as a supplement to standard chemotherapy $(2,44,45)$.

Glutamine. Glutamine is a non-essential amino acid with a special role in metabolism and nutrition. A number of key functions of glutamine in metabolism are shown in Table II (46). Being a precursor for nucleotide synthesis, glutamine availability is a key factor in cell growth. In the human body, glutamine availability is crucial for intestinal mucosal cells and also for immune-competent cells. When glutamine is used as an energy source, a high metabolic flow rate is present; furthermore a small fraction of that flux will be sufficient to dramatically increase the nucleotide synthesis rate. Therefore, many cells use glutamine as an energy substrate. It has been demonstrated that stressed cells have a particular preference for glutamine as an energy source. The role of glutamine for inter-organ transport is also well established (47).

An increasing number of clinical investigations have focused on the supplementation of specialized enteral and parenteral nutrition with the amino acid glutamine to improve the efficacy of nutritional support $(48,49)$.

Endogenous production of glutamine may become insufficient during critical illness. The shortage of glutamine is reflected as a decrease in plasma concentration, which is a prognostic factor for poor outcome in sepsis. Since glutamine is a precursor for nucleotide synthesis, rapidly dividing cells are most likely to suffer from a shortage. Therefore, exogenous glutamine supplementation is necessary (50). In particular, when i.v. nutrition is administered, extra glutamine supplementation becomes critical, as most current formulations for i.v. use do not contain any glutamine for technical reasons. The major portion of endogenously produced glutamine 
Table II. Glutamine functions.

\section{Precursor for DNA/RNA}

\section{Constituent for proteins}

Energy substrate for immunocompetent cells and enterocytes Substrate for gluconeogenesis

Precursor for glutamate in the brain - glutamate is an important excitatory neurotransmitter in the brain

Pathway for glutamate transport out of the brain

Via glutamate a precursor for glutathione, which is an antioxidant

Substrate for renal ammoniagenesis and acid-base regulation

comes from skeletal muscle. For patients who must remain for a long time in the intensive care unit (ICU), the muscle mass decreases rapidly, which leaves a tissue of diminishing size to maintain the export of glutamine. The metabolic and nutritional adaptation in long-staying ICU patients has been poorly studied and is one of the fields that requires more scientific evidence for clinical recommendations (47). To date, there is evidence to support the clinical use of glutamine supplementation in critically ill, in hematology and in oncology patients. Strong evidence is presently available for i.v. glutamine supplementation to critically ill patients on parenteral nutrition (51). This must be regarded as the standard of care. For patients on enteral nutrition, more evidence is required. Concerning the administration of glutamine, there are good arguments to use the measurement of plasma glutamine concentration for guidance. This may provide an indication for treatment as well as proper dosing. Most patients will have a normalized plasma glutamine concentration by adding $20-25 \mathrm{~g} / 24 \mathrm{~h}$. Furthermore, there are no reported adverse or negative effects attributable to glutamine supplementation (52).

Glutamine behaves as an essential amino acid in clinical settings where there is marked metabolic stress, such as that which occurs after HSCT (53). Glutamine supplementation in animals was found to reduce bacterial translocation and mucosal damage after chemotherapy or radiotherapy, making supplementation an attractive option for reducing post-transplant complications $(54,55)$. There has also been a lack of an objective tool for measuring mucosal barrier injury (MBI), not only of the mouth, but also of the digestive tract as a whole, despite the fact that MBI is the most frequent cause of morbidity associated with the myeloablative-conditioning treatment to prepare for an HSCT (56). Oral mucositis is relatively easy to recognize, whereas the detection of intestinal mucosal injury has relied essentially on non-specific symptoms, such as nausea, vomiting, diarrhea and abdominal cramps, which affect almost every HSCT recipient and do not necessary reflect MBI (57).

Oral and i.v. glutamine appear to have differing effects. There is no apparent reason why this should be the case. Glutamine (i.v.), when administered with TPN, produces improvement in gut function and less gut atrophy, suggesting that i.v. glutamine has local effects on the gut mucosa. Oral glutamine appears to have no effect on mortality, infections, time to neutrophil recovery or relapses. Oral glutamine may reduce mucositis (decreased average score and fewer days of opioids and trends for less days of mucositis and less severe mucositis) and Graft-vs.-host-disease (GVHD) (which may be due to mucositis being a risk factor for GVHD) $(58,59)$.

The administration of enteral or parenteral glutamine at doses of up to $40 \mathrm{~g} /$ day appears safe according to studies of patients receiving BMT and high-dose chemotherapy published to date (60). It is possible that glutamine is utilized as a growth factor in interactions between malignant tumors and chemotherapeutic drugs (61). Thus, carefully planned pharmacological studies may be required when large doses of glutamine are administered to patients receiving cytotoxic drugs. However, the available clinical data in cancer patients does not suggest that glutamine-supplemented nutrition enhances or induces tumor growth or worsens clinical outcomes. However, further study of long-term outcomes with other novel forms of therapy, is indicated to complement primarily short-term safety data available to date. Glutamine deserves further study to elucidate its interactions with methotrexate and to investigate its effects on autologous HSCT patients. Supplementation of glutamine should be considered in the design of future randomized, controlled clinical trials and in the metabolic support of individuals undergoing marrow transplantation and cancer $(60,62)$.

Probiotics and prebiotic oligosaccharides. Diarrhea is a common complication of enteral nutrition in cancer patients. For many years, fiber was extensively investigated for its role in preventing diarrhea; however, a more recent focus has been the investigation of specific fiber blends, including soluble fibers and prebiotics, for which there is now considerable quality evidence. Enteral nutrition may result in deleterious effects on gastrointestinal microbiota, including reductions in bifidobacteria and key butyrate producers. Their modulation by prebiotics has been confirmed in studies on healthy individuals, but convincing evidence in acutely ill patients is required (63).

The pathogenesis of diarrhea involves antibiotic prescription, enteropathogenic colonization and abnormal colonic responses, all of which involve an interaction with colonic microbiota. Alterations in the colonic microbiota have been identified in patients receiving enteral tube feeding, and these changes may be associated with the incidence of diarrhea. Preventing negative alterations in the colonic microbiota has therefore been investigated as a method of reducing the incidence of diarrhea. Probiotics and prebiotics may be effective because of their functions in modulating both the structure and composition as well as activities of both mucosa and microflora suppression of enteropathogenic colonization (stimulation of immune function and modulation of colonic metabolism) $(2,64)$. Randomized controlled trials of probiotics have produced contrasting results, although Saccharomyces boulardii has been shown to reduce the incidence of diarrhea in patients in the ICU receiving enteral tube feeding. Prebiotic fructo-oligosaccharides have been shown to increase the concentration of fecal bifidobacteria in healthy subjects consuming an enteral formula, although this finding has not yet been confirmed in patients receiving enteral TF. Furthermore, there are no clinical trials investigating the effect of a prebiotic alone on the incidence of diarrhea. Further trials of the efficacy of probiotics and prebiotics, alone and in 
combination, in preventing diarrhea in this patient group are warranted (64).

Probiotics and prebiotics may beneficially affect a series of GI functions by modulating both the structure and composition, as well as activities of both mucosa and microflora (64). Potential targets and expected benefits have been identified as reduced risk for metabolic syndrome and prevention of colorectal cancer (65).

The potential effects of probiotics and prebiotics in cancer are as follows $(64,66-68)$. i) Reversing the disruption of microbiota and improving resistance to colonization by pathogens: Gut microflora themselves act as barriers against invasion by pathogens. Chemotherapy profoundly disturbs floral balance in a manner that potentiates colonization by pathogens, and an efficient probiotic or prebiotic may favorably modify these changes, inhibiting overgrowth of potential pathogens that cause secondary infectious diarrhea following chemotherapy. ii) Beneficially modulating the immune system: Probiotics extensively influence intestinal innate/adaptive immunity and barrier function. Probiotics and prebiotics may also directly or indirectly modulate the inflammatory cytokine network. These probiotics and prebiotics appear to enhance the gut barrier and reduce inflammatory injury by rebalancing the proinflammatory/anti-inflammatory cytokine network. iii) Enhancing production of short-chain FAs (SCFAs): Fermentation of oligofructose and insulin increases production of SCFAs, primarily acetate, butyrate and propionate, in the gut. SCFAs, the main energy source for colonic mucosal enterocytes, play a central metabolic role in maintaining the epithelial cell barrier and in repairing mechanisms that are likely important for the prevention or resolution of inflammation. iv) Drug metabolism by intestinal microflora contributes to the pharmacological profile of various drugs: Probiotics and prebiotics may modulate the pharmacokinetics of anticancer drugs by altering the composition and metabolic activity of the microflora. More research is required to determine probiotic and prebiotic modulation of bacterial enzyme activity during chemotherapy.

Enteral nutrition in surgical cancer patients. During the last few years, the importance of the correct nutritional assessment as a part of the therapeutic process of human pathologies has a greatly increased relevance. Still more in oncology, such a relationship between nutritional assessment and a beneficial result of the therapeutic treatment has a fundamental importance (69). There is a clear correlation between the degree of malnutrition and increased risk of perioperative complications in cancer patients undergoing surgery. It is known from various studies that the value of a variety of nutrition status parameters for predicting risk of surgical complications is important (70). Nutrition support therapy should not be used routinely in patients undergoing major cancer operations (71). For surgical patients, practical information, such as weight loss or subjective global assessment, would provide a better basis for deciding whether or not to delay surgery. At least 10 days of nutritional support is recommended in severely malnourished patients before major digestive surgery. In non-severely malnourished patients, pre-operative oral immunonutrition is associated with a $50 \%$ decrease in post-operative complications. The benefit of immune-enhancing diets in severely malnourished patients remains to be proven. For patients undergoing radio- chemotherapy, dietary counseling is proposed. In cases of severely malnourished patients or if dietary counseling suffers a setback, EN should be recommended (72).

Total parenteral nutrition in surgical/non-surgical cancer patients. The maintenance or improvement of QoL, and the increase in the effectiveness of antitumor therapy and a reduction in side effects are further objectives in regards to cancer patients. Indications for TPN in tumor patients are essentially identical to those in patients with benign illnesses, with preference given to oral or enteral nutrition when feasible. A combined nutritional concept is preferred when oral or enteral nutrition, particularly exclusive artificial nutrition, is administered. The use of TPN as a general accompaniment to radiotherapy or chemotherapy is not indicated, but TPN is indicated in chronic severe radiogenic enteritis or after allogenic transplantation with pronounced mucositis or GVHD (73). General information regarding TPN application in oncology patients is shown in Table III (4).

The treatment aims for TPN in cancer patients (73) are as follows. i) TPN should stabilize the nutritional state and prevent or reduce progressive weight loss; ii) TPN should maintain or improve the QoL; iii) TPN may increase the effectivity and reduce the side effects of anticancer therapies.

The majority of cancer patients requiring long-term TPN are cachectic and hypophagic because of (subacute) intestinal obstruction due to peritoneal carcinomatosis. This condition is often associated with expansion of extracellular water volume, and an overzealous administration of glucose may easily precipitate a peritoneal effusion which consequently forces withdrawal of the intravenous nutrition (74).

In patients who are losing weight mainly because of an insufficient nutritional intake, artificial nutritional support should be provided to maintain nutritional status or at least prevent further nutritional deterioration. This may also contribute to the maintenance of QoL. Any such improvement in the nutritional status is usually modest and is most expected when weight loss is mainly due to hypophagia. In the presence of systemic inflammation, however, it appears to be extremely difficult to achieve whole body protein anabolism in cancer patients. In this situation, in addition to nutritional interventions, pharmacological efforts are recommended to modulate the inflammatory response (75). Patients who received the planned amounts of energy and nitrogen (given by vein when necessary) were found to exibit improved energy balance, increased body fat and greater maximum exercise capacity, in addition to prolonged survival when compared to patients randomized to support without TPN (76).

Enteral and parenteral nutrition confer a number of risks, including the physiologic stress and discomfort associated with the placement of a feeding tube or central line and complications involved in the placement or in nutrition. Infection is the most common complication of both types of nutritional support and occurs frequently with parenteral nutrition because of the high nutrient value of the infusion. Although usually easily treated, these infections often require hospitalization and insertion of a new catheter, and may lead to complications, such as subacute bacterial endocarditis. Other common complications of enteral and parenteral nutrition include metabolic problems, such as hyperglycemia and fluid, and electrolyte imbalances, diarrhea from enteral 
Table III. Summary of information regarding total parenteral nutrition.

Subject

General

Indications

Nutritional provision

Perioperative care

During non-surgical therapy

Incurable patients

Hematopoietic stem cell transplantation (HSCT)

Tumor growth
Recommendations

Nutritional assessment of all cancer patients should begin with tumor diagnosis and be repeated at every visit in order to initiate nutritional intervention early, before the general status is severely compromised.

Total daily energy expenditure in cancer patients may be assumed to be similar to healthy subjects, or 20-25 kcal/kg/day for bedridden and $25-30 \mathrm{kcal} / \mathrm{kg} /$ day for ambulatory patients. Therapeutic goals for TPN in cancer patients are the improvement of function and outcome by:

- preventing and treating undernutrition/cachexia,

- enhancing compliance with antitumor treatments,

- controlling certain adverse effects of antitumor therapies,

- improving QoL.

TPN is ineffective and probably harmful in non-aphagic oncological patients in whom there is no gastrointestinal reason for intestinal failure. TPN is recommended in patients with severe mucositis or severe radiation enteritis. Supplemental TPN is recommended in patients when inadequate food and enteral intake ( $<60 \%$ of the estimated energy expenditure) is anticipated for $>10$ days. TPN is not recommended if oral/enteral nutrient intake is adequate. In the presence of systemic inflammation, it is extremely difficult to achieve whole body protein anabolism in cancer patients. In this situation, in addition to nutritional interventions, pharmacological efforts are recommended to modulate the inflammatory response.

Preliminary data suggest a potential positive role of insulin (Grade C). There are no data on n-3 FAs. Peri-operative TPN is recommended in malnourished candidates for artificial nutrition, when EN is not possible.

Routine use of TPN during chemotherapy, radiotherapy or combined therapy is not recommended.

When patients are malnourished or are facing a period longer than 1 week of starvation and enteral nutritional support is not feasible, TPN is recommended. In intestinal failure, long-term TPN should be offered when i) enteral nutrition is insufficient, ii) expected survival due to tumor progression is longer than 2-3 months), iii) it is expected that PN can stabilize or improve performance status and QoL, and iv) the patient desires this mode of nutritional support.

There is probable benefit in providing incurable cancer patients with weight loss and reduced nutrient intake with 'supplemental' TPN. In HSCT patients, TPN should be reserved for those with severe mucositis, ileus or intractable vomiting. No clear recommendation can be made as to the time of introduction of TPN in HSCT patients. Its withdrawal should be considered when patients are able to tolerate approximately $50 \%$ of their requirements enterally. HSCT patients may benefit from glutamine-supplemented TPN.

Although TPN supplies nutrients to the tumor, there is no evidence that this has deleterious effects on the outcome. This consideration should therefore have no influence on the decision to feed a cancer patient when TPN is clinically indicated. feeding and hepatic abnormalities from parenteral feeding. In the terminally ill, both types of nutrition cause fluid overload, worsening edema or shortness of breath (77).

\section{Conclusion}

In conclusion, enteral or parenteral nutrition support decreases the catabolic rate of the patients, helping them withstand the side effects of the therapeutic measures, but do not reverse to anabolism. Terminally ill cancer patients who are refractory to the different therapeutic measures require palliative care. Nutrition is a basic human right and is conceived by the patient and his family, as well as by the medical community and human society, to be vital for survival. However, a group of patients exist who, although they are not candidates for antineoplastic therapy, do remain in good physical and mental condition with expected lifespans of 3 months or more, and who are suffering from conditions, such as intestinal obstruction or fistulas, which makes the preferred route of EN impossible. In this specific patient group, palliative parenteral nutrition should be considered. The decision should be taken after careful multidisciplinary discussion. The patient and caregivers should be aware that this is not a cancer-specific treatment and probably will not prolong the patient's life. 


\section{References}

1. Jones L, Watling RM, Wilkins S and Pizer B: Nutritional support in children and young people with cancer undergoing chemotherapy. Cochrane Database Syst Rev 7: 1-50, 2010.

2. Xue H, Sawyer MB, Wischmeyer PE and Baracos VE: Nutrition modulation of gastrointestinal toxicity related to cancer chemotherapy: from preclinical findings to clinical strategy. J Parenter Enteral Nutr 35: 74-90, 2011.

3. Shike M: Nutrition therapy for the cancer patient. Hematol Oncol Clin North Am 10: 221-234, 1996.

4. Bozzetti F, Arends J, Lundholm K, Micklewright A, Zurcher G and Muscaritoli M: ESPEN Guidelines on parenteral nutrition: non-surgical oncology. Clin Nutr 28: 445-454, 2009.

5. Morss S: Enteral and parenteral nutrition in terminally ill cancer patients: a review of the literature. Am J Hosp Palliat Care 23: 369-377, 2006

6. Muscaritoli M, Bossola R, Bellantone R and Fanelli FR: Therapy of muscle wasting in cancer: what is the future? Curr Opin Clin Nutr Metab Care 7: 459-466, 2004.

7. Simons JPFHA, Schols AM, Buurman WA and Wouters EF: Weight loss and low body cell mass in males with lung cancer: relationship with systemic inflammation, acute-phase response, resting energy expenditure, and catabolic and anabolic hormones. Clin Sci 97: 215-223, 1999.

8. Barber MD: The pathophysiology and treatment of cancer cachexia, Nutr Clin Pract 17: 203-209, 2002.

9. Tisdale MJ: Cachexia in cancer patients. Nat Rev Cancer 11: 862-871, 2002

10. Arends J: Metabolism in cancer patients. Anticancer Res 30: $1863-1868,2010$.

11. Van Cutsem E and Arends J: The causes and consequences of cancer-associated malnutrition. Eur J Oncol Nurs 9 (Suppl. 2): $51-63,2005$.

12. Jatoi A, Daly BD, Hughes VA, Dallal GE, Kehayias J and Roubenoff R: Do patients with nonmetastatic non-small cell lung cancer demonstrate altered resting energy expenditure? Ann Thorac Surg 72: 348-351, 2001.

13. Argilés JM, Olivan M, Busquets $\mathrm{S}$ and López-Soriano FJ: Optimal management of cancer anorexia-cachexia syndrome. Cancer Manag Res 22: 27-38, 2010.

14. Nitenberg $G$ and Raynard B: Nutritional support of the cancer patient: issues and dilemmas. Crit Rev Oncol Hematol 34: $137-168,2000$

15. Drudge-Coates L: Improving management of patients with advanced cancer. Patient Prefer Adherence 2: 415-424, 2010.

16. Marín Caro MM, Laviano A and Pichard C: Nutritional intervention and quality of life in adult oncology patients. Clin Nutr 26: 289-301, 2007.

17. Ströhle A, Zänker K and Hahn A: Nutrition in oncology: the case of micronutrients (Review). Oncol Rep 24: 815-828, 2010.

18. Marín Caro MM, Laviano A and Pichard C: Impact of nutrition on quality of life during cancer. Curr Opin Clin Nutr Metab Care 10: 480-487, 2007.

19. Tian J and Chen JS: Nutritional status and quality of life of the gastric cancer patients in Changle County of China. World J Gastroenterol 11: 1582-1586, 2005.

20. Gupta D, Lis CG, Granick J, Grutsch JF, Vashi PG and Lammersfeld CA: Malnutrition was associated with poor quality of life in colorectal cancer: a retrospective analysis. J Clin Epidemiol 59: 704-709, 2006.

21. Ravasco P, Monteiro-Grillo I, Vidal PM and Camilo ME: Cancer: disease and nutrition are key determinants of patients quality of life. Support Care Cancer 12: 246-252, 2004.

22. Petruson KM, Silander EM and Hammerlid EB: Quality of life as predictor of weight loss in patients with head and neck cancer. Head Neck 27: 302-310,2005.

23. Bower M, Jones W, Vessels B, Scoggins C and Martin R: Role of esophageal stents in the nutrition support of patients with esophageal malignancy. Nutr Clin Pract 25: 244-249, 2010.

24. Marín Caro MM, Gómez Candela C, Castillo Rabaneda R, Lourenço Nogueira T, García Huerta M and Loria Kohen V: [Nutritional risk evaluation and establishment of nutritional support in oncology patients according to the protocol of the Spanish Nutrition and Cancer Group]. Nutr Hosp 23: 458-468, 2008.

25. Mudigonda T and Mudigonda P: Palliative cancer care ethics: principles and challenges in the Indian setting. Indian J Palliat Care 16: 107-110, 2010 .
26. Maree JE and Wright SC: Palliative care: a positive outcome for cancer patients? Curationis 31: 43-49, 2008

27. Granda-Cameron C, Viola SR, Lynch MP and Polomano RC: Measuring patient-oriented outcomes in palliative care: functionality and quality of life. Clin J Oncol Nurs 12: 65-77, 2008.

28. Wiseman M: The treatment of oral problems in the palliative patient. J Can Dent Assoc 72: 453-458, 2006

29. Koretz RL: Should patients with cancer be offered nutritional support: Does the benefit outweigh the burden? Eur J Gastroenterol Hepatol 19: 379-382, 2007.

30. Arends J, Bodoky G, Bozzetti F, et al: ESPEN Guidelines on enteral nutrition: non-surgical oncology. Clin Nutr 25: 245-259, 2006.

31. ASPEN Board of Directors: Clinical Guidelines Task Force, Nutrition assessment - adults. J Parenter Enteral Nutr 26: 9-12, 2002.

32. Sax HC and Souba WW: Enteral and parenteral feedings Guidelines and recommendations. Med Clin North Am 77: 863-880, 1993

33. Hardman WE: Omega-3 FA to augment cancer therapy. J Nutr 132 (Suppl. 11): 3508, 2002.

34. Hardman WE: N-3 fatty acids and cancer therapy. J Nutr 134 (Suppl. 12): 3427-3430, 2004.

35. Caughey GE, Mantzioris E, Gibson RA, Cleland LG and James MJ: The effect on human tumor necrosis factor alpha and interleukin lbeta production of diets enriched in or fish oil. Am J Clin Nutr 63: 116-119, 1996.

36. Barber MD: Cancer cachexia and its treatment with fish-oilenriched nutritional supplementation. Nutrition 17: 751-755, 2001.

37. Moses AW, Slater C, Preston T, Barber MD and Fearon KC: Reduced total energy expenditure and physical activity in cachexia patients with pancreatic cancer can be modulated by an energy and protein dense oral supplement enriched with n-3 fatty acids. Br J Cancer 90: 996-1002, 2004.

38. Takatsuka H, Takemoto Y and Iwata N: Oral eicosapentaenoic acid for complications of bone marrow transplantation. Bone Marrow Transplant 28: 769-774, 2001.

39. Bruera E, Strasser F and Palmer JL: Effect of fish oil on appetite and other symptoms in patients with advanced cancer and anorexia/cachexia: a double-blind, placebo-controlled study. J Clin Oncol 21: 129-134, 2003.

40. Wigmore SJ, Ross JA, Falconer JS, Plester CE, Tisdale MJ, Carter DC and Fearon KC: The effect of polyunsaturated fatty acids on the progress of cachexia in patients with pancreatic cancer. Nutrition 12 (Suppl. 1): 27-30, 1996

41. Bauer JD and Capra S: Nutrition intervention improves outcomes in patients with cancer cachexia receiving chemotherapy - a pilot study. Support Care Cancer 213: 270-274, 2005.

42. Fearon KC, von Meyenfeldt MF, Moses AG, et al: Effect of a protein and energy dense n-3 fatty acid-enriched oral supplement on loss of weight and lean tissue in cancer cachexia: a randomised double blind trial. Gut 52: 1479-1486, 2003.

43. Read JA, Beale PJ, Volker DH, Smith N, Childs A and Clarke SJ: Nutrition intervention using an eicosapentaenoic acid (EPA)containing supplement in patients with advanced colorectal cancer. Effects on nutritional and inflammatory status: a phase II trial. Support Care Cancer 15: 301-307, 2007.

44. Spencer L, Mann C, Metcalfe M, Webb M, Pollard C and Spencer D: The effect of omega-3 FAs on tumour angiogenesis and their therapeutic potential. Eur J Cancer 45: 2077-2086, 2009.

45. Fritsche KL: Are omega-3 fatty acids effective in enhancing tumoricidal cell activity? J Nutr 135: 2916-2917, 2005.

46. Oudemans-van Straaten HM, Bosman RJ, Treskes M, van der Spoel HJ and Zandstra DF: Plasma glutamine depletion and patient outcome in acute ICU admissions. Intensive Care Med 27: 84-90, 2001.

47. Berg A, Rooyackers O, Norberg A and Wernerman J: Elimination kinetics of L-alanyl-L-glutamine in ICU patients. Amino Acids 29: 221-228, 2005.

48. Darmaun D: Role of glutamine depletion in severe illness. Diabetes Nutr Metab 13: 25-30, 2000.

49. Ziegler TR, Bazargan N and Galloway JR: Glutamine enriched parenteral nutrition; saving nitrogen and saving money? Clin Nutr 19: 375-377, 2000.

50. Novak F, Heyland DK, Avenell A, Drover JW and Su X: Glutamine supplementation in serious illness: a systematic review of the evidence. Crit Care Med 30: 2022-2029, 2002. 
51. Goeters C, Wenn A, Mertes N, et al: Parenteral L-alanyl-Lglutamine improves 6-month outcome in critically ill patients. Crit Care Med 30: 2032-2037, 2002.

52. Wernerman J: Clinical use of glutamine supplementation. J Nutr 138: 2040-2044, 2008.

53. Crowther M, Avenell A and Culligan DJ: Systematic review and meta-analyses of studies of glutamine supplementation in haematopoietic stem cell transplantation. Bone Marrow Transplant 44: 413-425, 2009.

54. Ziegler TR: Glutamine supplementation in cancer patients receiving bone marrow transplantation and high dose chemotherapy. J Nutr 131: 2578-2584, 2001.

55. Blijlevens NMA, Donnelly JP and De Pauw BE: Mucosal barrier injury: biology, pathology, clinical counterparts and consequences of intensive treatment for haematological malignancy: an overview. Bone Marrow Transplant 25: 1269-1278, 2000.

56. Elting LS, Cooksley C, Chambers M, Cantor SB, Manzullo E and Rubenstein EB: The burdens of cancer therapy: clinical and economic outcomes of chemotherapy-induced mucositis. Cancer 98: 1531-1539, 2003

57. Sonis ST, Oster G, Fuchs H, et al: Oral mucositis and the clinical and economic outcomes of hematopoietic stem-cell transplantation. J Clin Oncol 19: 2201-2205, 2001.

58. Kuskonmaz B, Yalcin S, Kucukbayrak O, et al: The effect of glutamine supplementation on hematopoietic stem cell transplan outcome in children: a case-control study. Pediatr Transplant 12: 47-51, 2008

59. Aquino VM, Harvey AR, Garvin JH, et al: A double-blind randomized placebo-controlled study of oral glutamine in the prevention of mucositis in children undergoing hematopoietic stem cell transplantation: a pediatric blood and marrow transplant consortium study. Bone Marrow Transplant 36: 611-616, 2005.

60. Ziegler TR: Glutamine supplementation in bone marrow transplantation. Br J Nutr 87 (Suppl. 1): 9-15, 2002.

61. Wilmore DW, Schloerb PR and Ziegler TR: Glutamine in the support of patients following bone marrow transplantation. Curr Opin Clin Nutr Metab Care 2: 323-327, 1999.

62. Arfons LM and Lazarus HM: Total parenteral nutrition and hematopoietic stem cell transplantation: an expensive placebo? Bone Marrow Transplant 36: 281-288, 2005.

63. Whelan K and Schneider SM: Mechanisms, prevention, and management of diarrhea in enteral nutrition. Curr Opin Gastroenterol 27: 152-159, 2011.

64. Whelan K: Enteral-tube-feeding diarrhoea: manipulating the colonic microbiota with probiotics and prebiotics. Proc Nutr Soc 66: 299-306, 2007.
65. De Preter V, Hamer HM, Windey K and Verbeke K: The impact of pre- and/or probiotics on human colonic metabolism: does it affect human health? Mol Nutr Food Res 55: 46-57, 2011.

66. Pagnini C, Corleto VD, Hoang SB, Saeed R, Cominelli F and Delle Fave G: Commensal bacteria and 'oncologic surveillance': suggestions from an experimental model. J Clin Gastroenterol 42: S193-S196, 2008

67. Pagnini C, Saeed R, Bamias G, Arseneau KO, Pizarro TT and Cominelli F: Probiotics promote gut health through stimulation of epithelial innate immunity. Proc Natl Acad Sci USA 107: 454-459, 2010

68. Roberfroid M, Gibson GR, Hoyles L, McCartney AL, Rastall R and Rowland I: Prebiotic effects: metabolic and health benefits. Br J Nutr 104 (Suppl. 2): 1-63, 2010.

69. Santacroce L, Leone D, Valenzano A, Luperto P, Bottalico L and Losacco T: Nutritional problems in the surgical patients with head and neck tumours. Literature review and personal experience. Clin Ter 156: 227-230, 2005 (In Italian).

70. Huhmann MB and August DA: Nutrition support in surgical oncology. Nutr Clin Pract 24: 520-526, 2009.

71. Huhmann MB and August DA: Review of American Society for Parenteral and Enteral Nutrition (ASPEN) clinical guidelines for nutrition support in cancer patients: nutrition screening and assessment. Nutr Clin Pract 23: 182-188, 2008.

72. Senesse P, Assenat E, Schneider S, Chargari C, Magné N and Azria D: Nutritional support during oncologic treatment of patients with gastrointestinal cancer: Who could benefit? Cancer Treat Rev 34: 568-575, 2008

73. Arends J, Zuercher G, Dossett A, Fietkau R, Hug M and Schmid I: Non-surgical oncology - Guidelines on arenteral nutrition. Ger Med Sci 18: 1-14, 2009.

74. Pironi L, Joly F, Forbes A, et al: Long-term follow-up of patients on home parenteral nutrition in Europe: implications for intestinal transplantation. Gut 60: 17-25, 2011.

75. Orrevall Y, Tishelman C, Herrington MK and Permert J: The path from oral nutrition to home parenteral nutrition: a qualitative interview study of the experiences of advanced cancer patients and their families. Clin Nutr 23: 1280-1287, 2004.

76. Braunschweig $\mathrm{C}$, Liang $\mathrm{H}$ and Sheean P: Indications for administration of parenteral nutrition in adults. Nutr Clin Pract 19 255-262, 2004.

77. Dy SM: Enteral and parenteral nutrition in terminally ill cancer patients: a review of the literature. Am J Hosp Palliat Care 23: 369-377, 2006. 